\title{
Blandford-Znajek process in vacuo and its holographic dual
}

\author{
Ted Jacobson* \\ Center for Fundamental Physics, University of Maryland, College Park, Maryland 20742, USA \\ Maria J. Rodriguez ${ }^{\dagger}$ \\ Max Planck for Gravitational Physics, Potsdam 14476, Germany \\ and Department of Physics, Utah State University, 4415 Old Main Hill Road, Utah 84322, USA
}

(Received 5 March 2019; published 11 June 2019)

\begin{abstract}
Blandford and Znajek discovered a process by which a spinning black hole can transfer rotational energy to a plasma, offering a mechanism for energy and jet emissions from quasars. Here we describe a version of this mechanism that operates with only vacuum electromagnetic fields outside the black hole. The setting, which is not astrophysically realistic, involves either a cylindrical black hole or one that lives in $2+1$ spacetime dimensions, and the field is given in simple, closed form for a wide class of metrics. For asymptotically anti-de Sitter black holes in $2+1$ dimensions, the holographic dual of this mechanism is the transfer of angular momentum and energy, via a resistive coupling, from a rotating thermal state containing an electric field to an external charge density rotating more slowly than the thermal state. In particular, the entropy increase of the thermal state due to Joule heating matches the Bekenstein-Hawking entropy increase of the black hole.
\end{abstract}

DOI: 10.1103/PhysRevD.99.124013

\section{INTRODUCTION}

Prodigious amounts of energy wind up in the rotation of spinning black holes. Although nothing can escape from inside a black hole, that energy can be extracted via a dynamo effect, the "Blandford-Znajek (BZ) process" [1], when a black hole is threaded by a magnetic field and surrounded by plasma. The mechanism of the BZ effect is a type of "Penrose process" [2]: near a spinning black hole is an "ergosphere" where it is impossible to remain stationary relative to a distant observer, because the local inertial frames are dragged around faster than the speed of light. A system in the ergosphere can carry negative values of the conserved global energy quantity, yet have positive ordinary energy relative to a local observer. (In this paper the word "energy" will refer to the globally conserved quantity, which agrees with "local energy" only far from the black hole, in its rest frame.) Total energy can therefore be globally conserved, while positive energy flows away and negative energy flows into the black hole.

\footnotetext{
jacobson@umd.edu

maria.rodriguez@usu.edu; maria.rodriguez@aei.mpg.de
}

Published by the American Physical Society under the terms of the Creative Commons Attribution 4.0 International license. Further distribution of this work must maintain attribution to the author(s) and the published article's title, journal citation, and DOI. Funded by SCOAP ${ }^{3}$.
Although the principles of the BZ process are fully understood (e.g., [3,4]), and it is well studied analytically, semianalytically, and numerically (e.g., [1,5-9]), it remains difficult to develop an intuitive picture of how the flow of electromagnetic energy and charge current is organized in space and how and where the negative energy originates. The difficulty is in part due to the three dimensionality, the number of different field quantities, and the complexity of the Kerr metric which describes the spacetime geometry of a spinning black hole. In an effort to boil down the process to a "toy model," we began looking for a version in a $2+1$-dimensional spacetime. We assumed that, as in the original BZ solution, the plasma is "force-free," i.e., that it has a (nonzero) charge 4-current density orthogonal to the field strength, but discovered that there is no such energy-extracting stationary axisymmetric solution (see Appendix A for a demonstration). Much to our surprise, however, we found that there is a purely electromagnetic version of the $\mathrm{BZ}$ process, in which plasma plays no role.

We begin here with cylindrical black hole $(\mathrm{BH})$ examples, since they are easier to visualize, and the analogy with the astrophysical case is closer. The $2+1$ version will then be obtained as a special case of the $3+1$ cylindrical one. Of particular interest is the spinning Banados-TeitelboimZanelli (BTZ) black hole background in $2+1$ dimensions [10], since it is an exact solution of general relativity with a negative cosmological constant, is locally maximally symmetric, and is related by AdS/CFT duality [11] to a thermal state of a two dimensional conformal field theory. Thus one 
can also examine the conformal field theory (CFT) dual of a $\mathrm{BZ}$ process. A similar idea was previously pursued in Ref. [12], with force-free plasma in the Kerr-anti-de Sitter (AdS) background, but no energy extracting solution was found. It was suggested there that this might be due to the existence of a globally timelike Killing vector outside the event horizon, but this implies only that positive values of the corresponding conserved quantity cannot be extracted, and this need not be the relevant energy. Indeed, the spinning BTZ black hole also has a globally timelike Killing vector, yet we find that positive energy extraction is possible.

\section{CYLINDRICAL BZ PROCESS}

In the $\mathrm{BZ}$ process [1] a stationary plasma, around a spinning black hole threaded by a magnetic field, radiates a Poynting flux of energy to infinity. To simplify the geometry, and to eliminate the need for charge outside the black hole, we enhance the axisymmetry to cylindrical symmetry. Since the azimuthal circumference grows as the cylindrical radius $r$, the radial component of the Poynting vector $\vec{E} \times \vec{B}$ must then fall as $1 / r$ to conserve energy. For example, if there is a uniform electric field parallel to the cylindrical axis, then the magnetic field must have an azimuthal, circular component that falls as $1 / r$. Ampère's law then implies that there must be an enclosed electric current parallel to the axis. In order for the Poynting flux to be radially outward, the flow of current must be opposite to the external electric field. The energy required to drive this current ends up in the outgoing Poynting flux. See Fig. 1 for an illustration of this scenario.

Consider this model first in flat, empty spacetime. If current flows only along the axis, the Poynting flux emerges from the axis. Of course a line source of Poynting flux does not much resemble the BZ process, so let us introduce a cylindrical black hole and hide the line source behind the event horizon. A solution essentially like the one just described can be placed on this black hole spacetime but, since nothing can escape from a black hole, we expect that energy cannot be extracted from the source behind the horizon. Indeed, if we try to construct such a solution, we find that the fields are singular on the horizon. In effect, the horizon becomes a singular source of energy, which again does not resemble the BZ process. However, if the black hole is spinning with angular velocity $\Omega_{H}$, and if there is a nonzero magnetic flux through the horizon that is related in a particular way to the electric field, the current, and $\Omega_{H}$, then outgoing Poynting flux exists with fields that are regular on the horizon. The electromagnetic dual of this solution has a uniform magnetic field parallel to the cylindrical axis, a radial electric flux through the horizon, and an azimuthal electric field. This reduces to a $2+1$ dimensional solution to Maxwell's equations when the direction along the axis is omitted. We turn now to a precise demonstration of these conclusions.

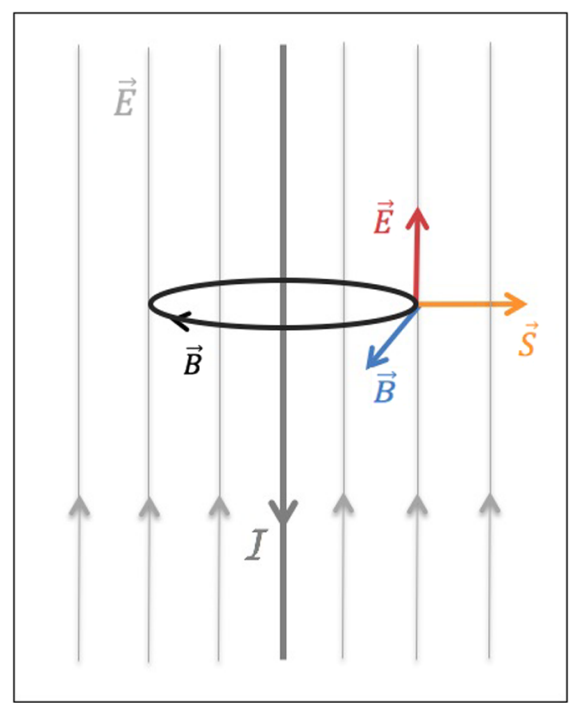

FIG. 1. A line current driven opposite to an ambient electric field emits a Poynting flux of energy. This field configuration corresponds to (2). Its generalization to any stationary axisymmetric spacetime (1), allowing for a radial component of the magnetic field, is given by (4). For spinning black cylinder spacetimes, the line current is behind the horizon, and the field is nonsingular on the horizon provided the Znajek condition (9) holds. Energy is then extracted from the black cylinder if there is magnetic flux through the horizon and $0<\Omega_{F}<\Omega_{H}$ (10). The nonzero black hole spin is what makes this extraction possible.

\section{A. Field configurations}

We adopt cylindrical spacetime coordinates $(t, r, \phi, z)$, and assume the spacetime line element takes the form

$$
d s^{2}=-\alpha^{2} d t^{2}+\alpha^{-2} d r^{2}+r^{2}(d \phi-\Omega d t)^{2}+d z^{2}
$$

where the functions $\alpha$ and $\Omega$ depend only on $r$, and we use units with $c=1$. The spacetime is then stationary and cylindrically symmetric: $d s^{2}$ is invariant under translation of $t, \phi$, and $z$. We refer to the $t$ coordinate as "time." This line element describes a wide class of spacetimes. If there is a radius $r_{H}$ where $\alpha\left(r_{H}\right)=0$, (1) describes a "black cylinder" or "black string," i.e., a black hole with a cylindrical horizon at the outermost root $r_{H}$. If $\Omega_{H}:=\Omega\left(r_{H}\right) \neq 0$, the black cylinder is spinning with angular velocity $\Omega_{H}$ and has an ergosphere where the time translation is spacelike, i.e., where $\alpha^{2}<r^{2} \Omega^{2}$. The BTZ black string metric [13] is conformal to (1). Since Maxwell's equations are conformally invariant, the solutions we discuss here are also solutions in the background of the BTZ black string.

To describe the electromagnetic field we employ the language of differential forms, which greatly simplifies computations, especially in curved spacetime. (For a concise review of differential forms and their application to electromagnetism, see Appendix A of [14].) The electromagnetic field strength 2 -form is denoted by $F$, 
an index-free notation for the antisymmetric tensor $F_{a b}$. (For example, $F=E d x \wedge d t+B d x \wedge d y$ describes an electric field strength $E$ in the $x$ direction and magnetic field strength $B$ in the $z$ direction, in flat spacetime with Cartesian coordinates.) Maxwell's equations are $d F=0$ (absence of magnetic monopoles and Faraday's law) and $d * F=J$ (Gauss and Ampère-Maxwell laws), where $d$ is the (metric independent) exterior derivative operator, $J$ is the current 3 -form, and $*$ is the Hodge dual operation that sends a $p$ form $\omega$ to the orthogonal $4-p$ form $* \omega$ with the same magnitude. We adopt the orientation of $d t \wedge d r \wedge$ $d \phi \wedge d z$ for defining the dual $\left(\omega \wedge \omega=\omega^{2} \epsilon\right.$, where $\omega^{2}$ is the squared norm of $\omega$ and $\epsilon$ is the unit 4-form with the adopted orientation). Note that the metric (1) is a sum of squares of 1-forms, hence those 1-forms are orthonormal, which facilitates the computation of duals and norms.

Let us begin with the simple example in flat spacetime,

$$
F=\left[\frac{I}{2 \pi r} d r-E d t\right] \wedge d z,
$$

where $I$ and $E$ are constants. This describes a uniform electric field in the $\hat{z}$ direction, together with a magnetic field circulating around the origin in planes of constant $t$ and $z$, sourced by a line current at $r=0$ flowing in the $-\hat{z}$ direction. The dual of the field (2) is

$$
* F=\frac{I}{2 \pi} d \phi \wedge d t-E r d \phi \wedge d r .
$$

It is clear by inspection that Maxwell's equations $d F=$ $0=d * F$ are satisfied (since $d^{2}=0$ and $d r \wedge d r=0$ ), so this is indeed a vacuum solution, except at the axis where $d \phi$ is singular. The field (2) is the wedge product of two 1 -forms, which implies that $F \wedge F=0$. Equivalently, the Lorentz-invariant scalar $\vec{E} \cdot \vec{B}$ vanishes, as it does for ideal plasmas since $\vec{E}$ vanishes in the rest frame of a perfect conductor. A field with this algebraic property is called "degenerate."

The field (2) can be easily generalized to the case of any background spacetime with a metric of the cylindrical form (1). Allowing also for a radial component of the magnetic field, the result is given by

$$
F=\left[\frac{I}{2 \pi r \alpha^{2}} d r+\psi_{z}\left(d \phi-\Omega_{F} d t\right)\right] \wedge d z,
$$

where $I, \psi_{z}$, and $\Omega_{F}$ are constants. The dual of (4) is

$$
\begin{aligned}
* F= & \frac{I}{2 \pi} d \phi \wedge d t \\
& +\psi_{z}\left[\frac{1}{r} d t+\frac{r\left(\Omega-\Omega_{F}\right)}{\alpha^{2}}(d \phi-\Omega d t)\right] \wedge d r .
\end{aligned}
$$

As with (2), Maxwell's equations are satisfied by inspection. (The functions $\alpha$ and $\Omega$ depend only on $r$, so that $d \alpha \wedge d r=0=d \Omega \wedge d r$.) The field (4) remains degenerate, since it is the wedge product of two 1-forms. To recover (2) from (4) one should set $\alpha=1$ and send $\psi_{z}$ to zero and $\Omega_{F}$ to infinity, with $E:=\psi_{z} \Omega_{F}$ held fixed. The constant parameters (and their notation) in (4) are directly analogous to functions that appear in standard treatments of stationary axisymmetric plasma magnetospheres (e.g., [14,15] and references therein).

The singularity on the axis carries a line current $I$ in the $-z$ direction, sourcing the azimuthal magnetic field, and a magnetic monopole line charge density $2 \pi \psi_{z}$, sourcing the radial magnetic field. ( $2 \pi \psi_{z}$ is the $z$ derivative of the magnetic flux through a cylinder ending at coordinate $z$.) The quantity $\Omega_{F}$ is the "angular velocity of the magnetic field lines," in the sense that the electric field vanishes in the local frame that rotates that way: $\left(\partial_{t}+\Omega_{F} \partial_{\phi}\right) \cdot F=0$. This is a standard concept in ideal plasma physics, where the plasma determines the preferred frame. It is meaningful here without the plasma only because the field is degenerate.

We are interested in the solution (4) on a rotating black hole background, but it is worth noting that it could also be terminated on a cylindrical conductor rotating with angular velocity $\Omega_{F}$.

\section{B. Energy extraction}

The Maxwell action is given by $-\frac{1}{2} \int F \wedge * F$, from which it follows that the conserved Noether current associated with the $t$-translation symmetry of the spacetime is [14]

$$
\mathcal{J}_{E}=-\left(\partial_{t} \cdot F\right) \wedge * F+\frac{1}{2} \partial_{t} \cdot(F \wedge * F) .
$$

As is usual, we call this conserved quantity simply energy. The outgoing energy flux is given by the integral of the 3 -form $\mathcal{J}_{E}$ over a surface of constant $r$, which by virtue of the conservation law is the same as the flux across any other surface of constant $r$. Replacing $\partial_{t} \rightarrow-\partial_{\phi}$ in (6), one obtains the angular momentum current $\mathcal{J}_{L}$ associated with the $\phi$-translation symmetry.

The energy current outward through a surface of constant $r$ arises from the part of $\mathcal{J}_{E}$ that contains no $d r$ factor [so the second term on the right-hand side of (6) does not contribute], which for the field (4) is given by

$$
\left.\mathcal{J}_{E}\right|_{r}=\frac{1}{2 \pi} \Omega_{F} \psi_{z} I d t \wedge d \phi \wedge d z .
$$

The outward flux of this current over a constant $r$ cylinder of length $\Delta z$ and time interval $\Delta t$ is $\Omega_{F} \psi_{z} I \Delta t \Delta z$, so the energy and angular momentum fluxes per unit proper length per unit $t$-coordinate time at any radius are given by

$$
\mathcal{E}_{r}=\Omega_{F} \psi_{z} I, \quad \mathcal{L}_{r}=\psi_{z} I .
$$


The outgoing energy flux is positive if $I$ has the same sign as $\Omega_{F} \psi_{z}$, which means that the current is opposite to the electric field. This corresponds to the situation described in the Introduction to this section, where the line current driven against the electric field produces an outgoing Poynting flux.

The line element (1) indicates that $\alpha d t$ and $d r / \alpha$ are unit 1 -forms, so that $d t$ and $d r / \alpha^{2}$ are both singular at $\alpha=0$ in a black cylinder spacetime. Therefore, the $I$ and $\Omega_{F}$ terms in the field (4) are both singular at the horizon. However, the forms $d t$ and $d r$ become proportional on the horizon, and their divergences can cancel. To analyze this divergence balancing act, one can either transform to a new coordinate system that is regular on the horizon, or construct scalars by contracting $F$ with a basis of finite vectors. One finds (see Appendix B) that a necessary condition for finiteness at the future horizon is

$$
I=2 \pi r_{H} \psi_{z}\left(\Omega_{H}-\Omega_{F}\right) .
$$

This condition is also sufficient, provided $\alpha^{2}$ has nonvanishing first derivative at $r_{H}$ (i.e., provided the black hole is not extremal). The relation (9) is known as the "Znajek condition" [16].

If the regularity condition (9) holds, then the outgoing energy flux (8) becomes

$$
\mathcal{E}_{r}=2 \pi\left(\psi_{z}\right)^{2} r_{H} \Omega_{F}\left(\Omega_{H}-\Omega_{F}\right) .
$$

If the black cylinder is not rotating $\left(\Omega_{H}=0\right)$, then the outward energy flux is always negative. This means that, as expected, while energy can flow inward, it cannot be extracted. However, if the black cylinder is rotating, and if $0<\Omega_{F}<\Omega_{H}$, then positive energy can be radiated outward provided there is a nonzero magnetic flux $\psi_{z}$ through the horizon. The energy flux formula (10) is precisely analogous to that for a force-free plasma on a Kerr black hole spacetime $[1,14,15]$.

The vacuum Maxwell equations are invariant under electric-magnetic duality, $F \rightarrow * F$, as is the energy momentum tensor and Noether current (6), so the dual (5) of the field (4) provides another solution that extracts energy from the black cylinder. The dual solution has a magnetic field in the $z$ direction and azimuthal and radial electric field components. The radial electric field is sourced by an electric line charge, and the azimuthal electric field can be sourced by either a magnetic monopole current or a magnetic flux line with flux proportional to time.

\section{2 + 1-DIMENSIONAL MODEL}

The dual field strength (5) has no $d z$ factor and is invariant under $z$ translations, so it descends to a solution $F_{3}:=*_{4} F$ in three-dimensional spacetime. The threedimensional Hodge dual $*_{3} F_{3}$ is a 1 -form, which is just the negative of the first factor of our original solution (4).
In the remainder of this article, we shall focus on this example. Maintaining the convention that the vector potential is an inverse length, in three spacetime dimensions the Maxwell action written at the beginning of Sec. II B should be multiplied by a constant with dimensions of length $1 / g^{2}$. We adopt units here with $g=1$.

Dualizing interchanges electric and magnetic quantities, so we change our notation for the parameters accordingly: $Q:=-2 \pi \psi_{z}$ is the electric charge of the solution, and $\dot{\Phi}:=-I$ is the $t$ derivative of the magnetic flux through a loop encircling the origin (or the magnetic monopole current). The constant $\Omega_{F}$ is now the "angular velocity of the electric field lines," that is, the angular velocity of the frame in which the magnetic field (which in $2+1$ dimensions is a spatial pseudoscalar) vanishes (presuming the vector $\partial_{t}+\Omega_{F} \partial_{\phi}$ is timelike, which is here the case for large enough $r$ ). The three-dimensional field and its dual are given by ${ }^{1}$

$$
\begin{gathered}
F_{3}=-\frac{\dot{\Phi}}{2 \pi} d \phi \wedge d t-\frac{Q}{2 \pi r} d t \wedge d r \\
-\frac{Q r\left(\Omega-\Omega_{F}\right)}{2 \pi \alpha^{2}}(d \phi-\Omega d t) \wedge d r, \\
*_{3} F_{3}=\frac{\dot{\Phi}}{2 \pi \alpha^{2} r} d r+\frac{Q}{2 \pi}\left(d \phi-\Omega_{F} d t\right) .
\end{gathered}
$$

The Znajek horizon regularity condition (9) in the present notation is $\dot{\Phi}=Q r_{+}\left(\Omega_{H}-\Omega_{F}\right)$. The dual of the regular field is thus

$$
*_{3} F_{3}=\frac{Q}{2 \pi}\left[\frac{r_{+}\left(\Omega_{H}-\Omega_{F}\right)}{\alpha^{2} r} d r+d \phi-\Omega_{F} d t\right] .
$$

This solution can be placed in particular on the rotating BTZ black hole background [10], which is a three-dimensional solution of Einstein's equations with a negative cosmological constant, $\Lambda_{3}=-\ell^{-2}$. The corresponding line element (in Boyer-Lindquist-like coordinates) is (1) without $d z^{2}$, and with

$$
\alpha^{2}=\frac{\left(r^{2}-r_{+}^{2}\right)\left(r^{2}-r_{-}^{2}\right)}{r^{2} \ell^{2}}, \quad \Omega=\frac{r_{-} r_{+}}{r^{2} \ell},
$$

where $r_{-}$and $r_{+}$are the inner and outer horizon radii, respectively, with $0<r_{-}<r_{+}$. On this spacetime, the invariant square of the field strength is

$$
F_{3}^{2}=\frac{Q^{2}}{4 \pi^{2}} \frac{\ell^{2} \Omega_{F}^{2}-1}{r^{2}-r_{-}^{2}} .
$$

In the energy extracting case $\left(0<\Omega_{F}<\Omega_{H}\right)$ we have $\ell \Omega_{F}<\ell \Omega_{H} \leq 1$, so this field is electric dominated

\footnotetext{
${ }^{1}$ All stationary, axisymmetric vacuum solutions to Maxwell's equations in a 2+1-dimensional spacetime (with the same symmetry) have this form.
} 


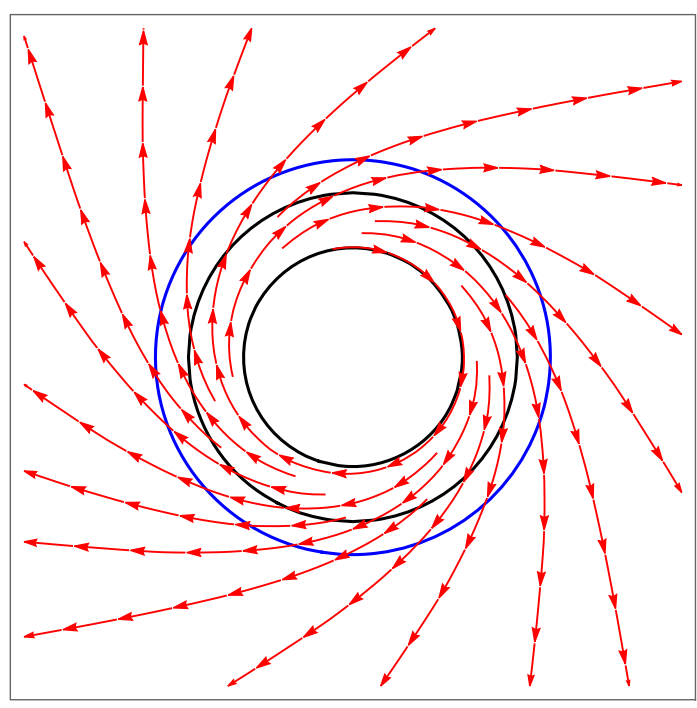

FIG. 2. Electric field lines for the field (13), with $r_{+}=0.3$, $r_{-}=0.2$, and $\Omega_{F}=\Omega_{H} / 2=1 / 3$, in units with $\ell=1$. As explained in Appendix $\mathrm{C}$, the field lines are intersections of the electric field sheets with a surface of constant ingoing Kerr coordinate $v$, plotted with $r$ and $\bar{\phi}$ treated as polar coordinates on the Euclidean plane. The field lines for $r<r_{-}$are not plotted, since $F_{3}$ is singular at $r_{-}$.

everywhere outside the inner horizon and is singular at the inner horizon. (For the case $\Omega_{F}=\Omega_{H}$ this singularity has been previously noted [17].) In Appendix $C$ we express the BTZ metric and the electromagnetic field in terms of ingoing Kerr coordinates $(v, r, \bar{\phi})$, which are regular everywhere except at $r=0$. The electric field lines on a surface of constant $v$ are plotted in Fig. 2.

\section{A. CFT dual}

We now turn to the AdS/CFT dual interpretation of the vacuum BZ process on the spinning BTZ black hole background. A number of previous studies have employed this duality with Einstein-Maxwell theory in three spacetime dimensions, sometimes with other fields included (see e.g. [18-23]), to study conductivity properties of the dual $1+1$-dimensional system. The black hole is dual to a thermal state of a CFT at the Hawking temperature, rotating with angular velocity $\Omega_{H}$ [24]. To extract rotational energy from this thermal state, it must be coupled to another system, and that coupling is described by the boundary conditions on the electromagnetic field. In the limit of large radius $r$, the field $F_{3}(11)$ can be derived (as $F_{3}=d A_{3}$ ) from the potential

$$
A_{3} \stackrel{r \rightarrow \infty}{\longrightarrow} \frac{\dot{\Phi}}{2 \pi} t d \phi-\frac{Q}{2 \pi} \ln r\left(d t-\ell^{2} \Omega_{F} d \phi\right)
$$

which corresponds, via the AdS/CFT dictionary [19,25], to a boundary CFT current $j$ and gauge field $a$,

$$
j=\frac{Q}{2 \pi \ell}\left(\partial_{t}+\Omega_{F} \partial_{\phi}\right), \quad a=\frac{\dot{\Phi}}{2 \pi} t d \phi .
$$

One dynamically consistent boundary condition fixes $j$ [25], which fixes the black hole charge and the asymptotic magnetic field. The transfer of energy between the CFT and the (unspecified) degrees of freedom carrying the current then entails Ohmic dissipation.

The conductivity $\sigma$ of the CFT is the ratio of the current to the electric field, figured in the rest frame of the thermal state. That is, $\sigma=(s \cdot j) /(u \cdot s \cdot f)$, where $f=d a=$ $(\dot{\Phi} / 2 \pi) d t \wedge d \phi$ is the boundary electromagnetic field strength, $u=\gamma\left(\partial_{t}+\Omega_{H} \partial_{\phi}\right)$ is the velocity 2-vector of this rotating frame, and $s=\gamma\left(\ell \Omega_{H} \partial_{t}+\ell^{-1} \partial_{\phi}\right)$ is the orthogonal, spatial unit 2-vector (with respect to the boundary metric, $\left.d s^{2}=-d t^{2}+\ell^{2} d \phi^{2}\right)$, with $\gamma=\left(1-\ell^{2} \Omega_{H}^{2}\right)^{-1 / 2}$. After using the Znajek horizon regularity condition, the dependence on the electromagnetic field parameters drops out, and we find

$$
\sigma=\frac{\gamma \ell}{r_{+}}=\frac{1}{\gamma \kappa \ell}=\frac{\hbar}{2 \pi \gamma T_{H}},
$$

where $\kappa$ is the surface gravity of the BTZ black hole, and $T_{H}=\hbar \kappa / 2 \pi$ is the Hawking temperature. In the nonrotating limit, $\sigma$ is inversely proportional to the Hawking temperature, in agreement with a prior result [22]. The conductivity depends on angular momentum of the black hole only via the Lorentz boost factor $\gamma$, and $\gamma T_{H}$ is the temperature in the corotating frame of the thermal state [26], so in fact the rotating conductivity is the same as in the nonrotating case when expressed in terms of the corotating temperature.

\section{B. Black hole evolution}

The response to weak fluxes of electromagnetic energy and angular momentum can be determined using the laws of black hole mechanics (which are derived from Einstein's equation). The flux formula (8) shows that $d E=\Omega_{F} d L$ for the electromagnetic fluxes of energy and angular momentum, and conservation laws imply that infinitesimal changes of the black hole mass $M$ and angular momentum $J$ are related in the same way, $d M=\Omega_{F} d J$. The first law of black hole mechanics [27] gives $d M-\Omega_{H} d J=(\kappa / 8 \pi G) d A$, where $\kappa$ and $A$ are the surface gravity and area of the horizon, respectively, hence $\left(\Omega_{F}-\Omega_{H}\right) d J=(\kappa / 8 \pi G) d A$. The second law of black hole mechanics [28] states that $d A \geq 0$, in processes with matter satisfying the null positive energy condition (such as electromagnetic fields). If $\Omega_{F}<\Omega_{H}$, the black hole, therefore, spins down, in which case it loses or gains mass if $\Omega_{F}$ is positive or negative, respectively. If instead $\Omega_{F}>\Omega_{H}$, the black hole spins up and gains mass. If the asymptotic electric and magnetic fields are held fixed, then $\Omega_{F}$ is fixed, and $\Omega_{H}$ evolves until it 
exponentially approaches the condition $\Omega_{H}=\Omega_{F}$, for which the fluxes vanish.

The black hole entropy $S_{\mathrm{BH}}=A / 4 \hbar G$ evolves as

$\dot{S}_{\mathrm{BH}}=\frac{\dot{M}-\Omega_{H} \dot{J}}{T_{H}}=\frac{\left(\Omega_{F}-\Omega_{H}\right) \dot{J}}{T_{H}}=\frac{\dot{\Phi} Q\left(\Omega_{H}-\Omega_{F}\right)}{2 \pi T_{H}}$,

where the overdot signifies a derivative with respect to the Killing time $t$, and $T_{H}=\hbar \kappa / 2 \pi$ is the Hawking temperature. [The first equality invokes the first law of black hole mechanics, while the second and third make use of (8) with the notational change indicated in the paragraph containing (11).]

The rate of entropy generation per unit spacetime volume, due to Ohmic dissipation in the holographic dual field theory process, is given by the rate of energy dissipation divided by the temperature in the corotating frame of the thermal state. The energy dissipation rate is the inner product of the electric field with the charge current in that frame, hence the total rate of entropy generation is

$$
\dot{S}_{\mathrm{FT}}=\frac{(u \cdot j \cdot F) 2 \pi \ell}{\gamma T_{H}} .
$$

This agrees with (19) when the previously given expressions for $u, j$, and $F$ are inserted. (This is special case of a very general relation between boundary and black hole entropy generation that has been established in [29].) It is amusing to note that this dual description is a perfect electrical analogy for the mechanical analogy originally described by Blandford and Znajek [1]. That analogy involved a thermally conducting disk spinning with angular velocity $\Omega_{H}$, coupled by friction to a concentric, thermally insulating ring spinning with a smaller angular velocity.

\section{DISCUSSION}

We have thus far treated the electromagnetic field as a test field on the background of a spinning BTZ black hole background and inferred adiabatic evolution from the conservation laws. To more fully probe the duality one should solve the coupled Einstein-Maxwell equations. A similar study was carried out in [22] for the case of electromagnetic energy flowing into a nonrotating black hole, allowing the nonlinear conductivity of the dual field theory to be found. In our case, a first step would be to use for the background the charged, spinning black hole solution [17,30], which also has a magnetic field with $\Omega_{F}=\Omega_{H}$ in our notation. This is the solution to which the black hole would relax, and the azimuthal electric field alone could be treated as a test field. The next step would be to attempt to find an Einstein-Maxwell solution including the azimuthal electric field, which could describe nonlinear effects in the conductivity. Another avenue to investigate would be alternate boundary conditions at infinity, which can modify the nature of the dual field theory degrees of freedom and the external source that couples to them $[19,21,25]$.

\section{ACKNOWLEDGMENTS}

We thank S. Hartnoll, G. Horowitz, N. Iqbal, K. Jensen, D. Marolf, A. Speranza, and S. Theisen for helpful discussions and correspondence. This research was initiated at the Peyresq Physics 21 meeting and partially supported there by OLAM. T. J. was also supported in part by NSF Grants No. PHY-1407744 and No. PHY1708139 at the University of Maryland, and by Perimeter Institute for Theoretical Physics. Research at Perimeter Institute is supported by the Government of Canada through Industry Canada and by the Province of Ontario through the Ministry of Research and Innovation. The work of M. J. R. was supported by the Max Planck Gesellschaft through the Gravitation and Black Hole Theory Independent Research Group and by NSF Grant No. PHY-1707571 at Utah State University.

\section{APPENDIX A: STATIONARY AXISYMMETRIC FORCE-FREE SOLUTIONS IN 2+1 DIMENSIONS}

In three spacetime dimensions, the force-free condition $F_{a b} j^{a}=0$ is equivalent to the condition

$$
d * F=\zeta F,
$$

for some function $\zeta$. If $F$ is stationary and axisymmetric then $* F$ has the form $* F=\alpha(r) d t+\beta(r) d \phi+\gamma(r) d r$, so $d * F=\alpha^{\prime} d r \wedge d t+\beta^{\prime} d r \wedge d \phi$. The force-free condition (A1) thus implies that either the charge current vanishes, or $F \propto \alpha^{\prime} d r \wedge d t+\beta^{\prime} d r \wedge d \phi$, in which case $F$ has no $d t \wedge$ $d \phi$ term (i.e., no azimuthal electric field). But this implies that the energy current

$$
\mathcal{J}_{E}=-\left(\partial_{t} \cdot F\right) \wedge * F+\frac{1}{2} \partial_{t} \cdot(F \wedge * F)
$$

has no $d t \wedge d \phi$ term, so there is no radial energy flux. (Equivalently, the Poynting vector has no radial component.)

\section{APPENDIX B: DERIVATION OF THE ZNAJEK CONDITION}

To characterize the regularity condition for the field strength

$$
F=\left[\frac{I}{2 \pi r \alpha^{2}} d r+\psi_{z}\left(d \phi-\Omega_{F} d t\right)\right] \wedge d z
$$

on the horizon, one can either transform to a coordinate system that is regular on the horizon or characterize $F$ by the scalars obtained when contracting it with a basis of regular vectors. Here we use the second method. For three of the four basis vectors we can use the Killing vectors, $\partial_{t}$, $\partial_{\phi}$, and $\partial_{z}$, which are all tangent to the horizon. (Note that, 
although the coordinates $t$ and $\phi$ are singular on the horizon of a spinning black hole, the vectors $\partial_{t}$ and $\partial_{\phi}$ are the time translation and rotation Killing vectors and, regardless of what coordinate system is used, these vectors are regular on the horizon.) The contractions of the three Killing vectors with $F$ are finite as long as the constants $\psi_{z}$ and $\Omega_{F}$ are finite. For the fourth regular basis vector we use the 4-velocity $k$ of null $(k \cdot k=0)$, zero angular momentum $\left(k \cdot \partial_{\phi}=0\right)$, and zero longitudinal momentum $\left(k \cdot \partial_{z}=0\right)$ geodesics, with the affine parameter fixed by the choice $k \cdot \partial_{t}=-1$. These conditions (which are conserved along the geodesic) determine $k$ uniquely up to the choice of whether the geodesic is ingoing or outgoing. The ingoing one is

$$
k=\alpha^{-2}\left(\partial_{t}+\Omega \partial_{\phi}\right)-\partial_{r}
$$

The additional nonzero scalar that can be formed using $k$ is

$$
k \cdot \partial_{z} \cdot F=\frac{\psi_{z}\left(\Omega-\Omega_{F}\right)-I / 2 \pi r}{\alpha^{2}} .
$$

A necessary condition for finiteness at the future horizon is therefore

$$
I=2 \pi r_{H} \psi_{z}\left(\Omega_{H}-\Omega_{F}\right) .
$$

This is also sufficient, provided $\left(\alpha^{2}\right)_{,}$is nonvanishing at $r_{H}$, as is the case as long as the black hole is not extremal.

\section{APPENDIX C: INGOING KERR COORDINATES FOR THE BTZ BLACK HOLE}

The spinning BTZ black hole metric is given in BoyerLindquist coordinates by (1) less the $d z^{2}$ term and with $\alpha$ and $\Omega$ given by (14). The coordinates $t$ and $\phi$ are singular at the horizon in this spacetime. In their place, we can introduce new coordinates $v=t+f(r)$ and $\bar{\phi}=$ $\phi+g(r)$, which will be regular if the functions $f(r)$ and $g(r)$ are defined so that $v$ and $\bar{\phi}$ are constant on the infalling, zero angular momentum null geodesics, whose affine 4 -velocity $k$ is given by (B2). The requirements $k \cdot d v=0$ and $k \cdot d \phi=0$ then impose $f^{\prime}=\alpha^{-2}-1$ and $g^{\prime}=\alpha^{-2}$, respectively, hence we have

$$
d v=d t+\frac{1}{\alpha^{2}} d r, \quad d \bar{\phi}=d \phi+\frac{\Omega}{\alpha^{2}} d r .
$$

In terms of these regular differentials, the metric takes the form

$$
d s^{2}=-\alpha^{2} d v^{2}+2 d v d r+r^{2}(d \bar{\phi}-\Omega d v)^{2},
$$

which is regular except at $r=0$. This is called the ingoing Kerr coordinate system.

When expressed using these regular coordinates, the dual field (12) takes the form

$*_{3} F_{3}=\frac{Q}{2 \pi}\left[\frac{\ell\left(r_{-}+\ell \Omega_{F} r\right)}{\left(r+r_{+}\right)\left(r^{2}-r_{-}^{2}\right)} d r+d \bar{\phi}-\Omega_{F} d v\right]$.

(We write the dual 1-form since it is simpler than the field strength 2 -form $F_{3}$.) Since there is no globally defined, natural time coordinate relative to which one might define the electric field vector, we capture the "shape" of the field (C3) as follows. The kernel of $*_{3} F_{3}$ at each point is twodimensional and is timelike outside the inner horizon since $F_{3}$ is electric dominated there (15). Since there is no charge current, $*_{3} F_{3}$ is closed $\left(d *_{3} F_{3}=0\right)$, so this distribution of planes is integrable, i.e., they are tangent to two-dimensional submanifolds, which can be called the "electric field sheets" (see [14] for a discussion of the magnetic case in four spacetime dimensions). The intersection of an electric field sheet with a spacelike surface is an electric field line on that surface. However, since we do not have preferred spacelike surfaces in the BTZ spacetime, we employ the intersection with a constant $v$ null surface to generate a picture of the electric field lines. They are tangent to a vector field $X$ satisfying $X \cdot *_{3} F_{3}=0=X \cdot d v$. One more condition is needed to specify the magnitude of $X$, although this magnitude will not affect the field lines. If we choose for this condition $X \cdot d r=1$, then

$$
X=\partial_{r}-\frac{\ell\left(r_{-}+\ell \Omega_{F} r\right)}{\left(r+r_{+}\right)\left(r^{2}-r_{-}^{2}\right)} \partial_{\bar{\phi}}
$$

In Fig. 2 we plot the streamlines of this vector field, treating $r$ and $\bar{\phi}$ as polar coordinates on the Euclidean plane.
[1] R. D. Blandford and R. L. Znajek, Electromagnetic extraction of energy from Kerr black holes, Mon. Not. R. Astron. Soc. 179, 433 (1977).

[2] R. Penrose, Gravitational collapse: The role of general relativity, Riv. Nuovo Cimento 1, 252 (1969); "Golden Oldie": Gravitational collapse: The role of general relativity, Gen. Relativ. Gravit. 34, 1141 (2002).
[3] I. Okamoto, Electromagnetic extraction of energy from Kerr black holes, Publ. Astron. Soc. Jpn. 58, 1047 (2006).

[4] J.-P. Lasota, E. Gourgoulhon, M. A. Abramowicz, A. Tchekhovskoy, and R. Narayan, Extracting black-hole rotational energy: The generalized Penrose process, Phys. Rev. D 89, 024041 (2014). 
[5] S. E. Gralla, A. Lupsasca, and M. J. Rodriguez, Electromagnetic jets from stars and black holes, Phys. Rev. D 93, 044038 (2016).

[6] A. Nathanail and I. Contopoulos, Black hole magnetospheres, Astrophys. J. 788, 186 (2014).

[7] S.S. Komissarov, Direct numerical simulations of the Blandford-Znajek effect, Mon. Not. R. Astron. Soc. 326, L41 (2001).

[8] S. S. Komissarov and J. C. McKinney, Meissner effect and Blandford-Znajek mechanism in conductive black hole magnetospheres, Mon. Not. R. Astron. Soc. 377, L49 (2007).

[9] J. C. McKinney, General relativistic magnetohydrodynamic simulations of jet formation and large-scale propagation from black hole accretion systems, Mon. Not. R. Astron. Soc. 368, 1561 (2006).

[10] M. Banados, C. Teitelboim, and J. Zanelli, The Black Hole in Three-Dimensional Space-Time, Phys. Rev. Lett. 69, 1849 (1992).

[11] J. M. Maldacena, The large N limit of superconformal field theories and supergravity, Int. J. Theor. Phys. 38, 1113 (1999); Erratum, Adv. Theor. Math. Phys. 2, 231(E) (1998).

[12] X. Wang and A. Ritz, Kerr-AdS black holes and force-free magnetospheres, Phys. Rev. D 89, 106011 (2014).

[13] R. Emparan, G. T. Horowitz, and R. C. Myers, Exact description of black holes on branes. 2. Comparison with BTZ black holes and black strings, J. High Energy Phys. 01 (2000) 021.

[14] S. E. Gralla and T. Jacobson, Spacetime approach to forcefree magnetospheres, Mon. Not. R. Astron. Soc. 445, 2500 (2014).

[15] D. MacDonald and K. S. Thorne, Black-hole electrodynamics-An absolute-space/universal-time formulation, Mon. Not. R. Astron. Soc. 198, 345 (1982).

[16] R. L. Znajek, Black hole electrodynamics and the Carter tetrad, Mon. Not. R. Astron. Soc. 179, 457 (1977).
[17] C. Martinez, C. Teitelboim, and J. Zanelli, Charged rotating black hole in three space-time dimensions, Phys. Rev. D 61, 104013 (2000).

[18] D. Maity, S. Sarkar, N. Sircar, B. Sathiapalan, and R. Shankar, Properties of CFTs dual to charged BTZ black-hole, Nucl. Phys. B839, 526 (2010).

[19] K. Jensen, Chiral anomalies and AdS/CMT in two dimensions, J. High Energy Phys. 01 (2011) 109.

[20] J. Ren, One-dimensional holographic superconductor from $\mathrm{AdS}_{3} / \mathrm{CFT}_{2}$ correspondence, J. High Energy Phys. 11 (2010) 055.

[21] T. Faulkner and N. Iqbal, Friedel oscillations and horizon charge in 1D holographic liquids, J. High Energy Phys. 07 (2013) 060.

[22] G. T. Horowitz, N. Iqbal, and J. E. Santos, Simple holographic model of nonlinear conductivity, Phys. Rev. D 88, 126002 (2013).

[23] P. Chaturvedi and G. Sengupta, Rotating BTZ black holes and one dimensional holographic superconductors, Phys. Rev. D 90, 046002 (2014).

[24] S. W. Hawking, C. J. Hunter, and M. Taylor, Rotation and the AdS/CFT correspondence, Phys. Rev. D 59, 064005 (1999).

[25] D. Marolf and S. F. Ross, Boundary conditions and new dualities: Vector fields in AdS/CFT, J. High Energy Phys. 11 (2006) 085.

[26] V. P. Frolov and K. S. Thorne, Renormalized stress-energy tensor near the horizon of a slowly evolving, rotating black hole, Phys. Rev. D 39, 2125 (1989).

[27] J. M. Bardeen, B. Carter, and S. W. Hawking, The four laws of black hole mechanics, Commun. Math. Phys. 31, 161 (1973).

[28] S. W. Hawking, Black holes in general relativity, Commun. Math. Phys. 25, 152 (1972).

[29] Y. Tian, X.-N. Wu, and H.-B. Zhang, Holographic entropy production, J. High Energy Phys. 10 (2014) 170.

[30] G. Clement, Spinning charged BTZ black holes and selfdual particle-like solutions, Phys. Lett. B 367, 70 (1996). 